\title{
ANISOTROPIC CREEP AND DAMAGE IN STRUCTURAL ELEMENTS UNDER CYCLIC LOADING*
}

\author{
D. V. Breslavskii, V. A. Metelev, \\ and O. K. Morachkovskii
}

UDC 539.3

\begin{abstract}
A method is described to calculate creep and damage in structural elements under cyclic loading. Materials exhibiting anisotropic creep-damage behavior are considered. The results of calculating the deformation and fracture of structural elements at a plane stress state are presented.
\end{abstract}

Keywords: creep, damage, cyclic loading, tensor parameter of damage, finite element method, plate with a hole.

Introduction. Metallic composite materials are widely used in industry. The anisotropy of creep and creep rupture strength properties, and a significant material inhomogeheity are typical of new materials as well as of those structural materials which have a wide use in aeronautical engineering and power engineering industry. In case of structural elements, this is due to their manufacture technology and stress state inhomogeneity. Many structures, especially those in aeronautical engineering and power engineering industry, operate under conditions of combined action of static loads and cyclically varying temperatures and stresses. In this connection, constitutive equations derived for the calculation analysis should take into account the factors, such as the initial and strain-induced anisotropy, anisotropy of accumulated hidden damage properties, effects of cyclic loadings and heating. Based on the LM-hardness method or other methods of hardness testing, the homogeneity coefficient can be used as a measure of damage [1].

At present, the fracture (macroscopic discontinuity of the body due to its exposure to external factors) is studied with consideration of the hidden damage processes [2-8]. The investigation of the hidden damage (nucleation and propagation of microdefects dispersed in the bulk of the body) is carried out using the damage mechanics methods and theories - a dynamically developing branch of the modern mechanics of a deformable solid. Currently, the problems of computer simulation of crack growth and creep rupture using different measures of damage have been discussed in many works [6-10]. Continuum damage mechanics is based on that the variation in material mechanical properties with time can be phenomenologically interpreted as a result of the accumulation of damages, different defects, micropores, and cracks. If damages reach a dangerous level, a fracture occurs.

Much attention has also been given to the computer simulation of the processes of deformation, damage and continuum damage in nonlinear materials and structures [2-10] with the use of the scalar and tensor measure of damage. Two main directions in the development of continuum damage mechanics can be distinguished [2-10]. The first is the analysis of the interrelation between the processes of damage accumulation and stress-strain state evolution caused by accumulation of scattered damages and the second is the computer simulation of damage and implementation of various measures into CAE systems. Special attention is paid to the introduction of the tensor measures of damage which reflect the anisotropic nature of damage accumulation in a solid body.

The prediction of the strength and life of structural elements suggests, firstly, specifying the mathematical model of material nonlinear deformation and damage, and then going to the finite element solutions of problems in

* Paper presented at the 5th International Scientific Conference "Problems of Dynamics and Strength in Turbomachinery" (Turbo-2014), May 27-30, 2014, Kiev, Ukraine.

National Technical University "KhPI," Kharkov, Ukraine. Translated from Problemy Prochnosti, No. 2, pp. 21 - 29, March - April, 2015. Original article submitted January 12, 2015. 
determination of the stress-strain state characteristics of structural elements. The existing computational methods, including those implemented in modern software packages, do not allow the consideration of the initial anisotropy and inhomogeneity of the body in the case of creep and creep rupture strength.

At present, many approaches to the simulation of anisotropic damage have been proposed. A sufficiently complete list of works dealing with the tensor measures of damage is given in [11]. The references to later works and the presentation of the anisotropic damage in terms of the second-order damage tensor are given in [12-15]. As a whole, literary sources dealing with the tensor measures of damage are so numerous that deserve a separate discussion. Next, the first two of the above trends will be considered.

The paper presents the method of calculating the dynamic creep and damage in structural elements made of materials with an anisotropic creep damage behavior.

Anisotropic Creep-Damage Models. Next, we employ the method presented earlier [12] for isotropic materials. We shall use the tensor relations of the incremental creep theory, which will be transformed into the dynamic creep relations of anisotropic materials with the help of the asymptotic small-parameter expansion and subsequent averaging over the period of load variation.

Consider a creep law including damage for anisotropic materials that satisfies the general tensor-linear relationships in the principal axes of symmetry and damage of the anisotropic body. Assume that the creep damage in the anisotropic material is determined by an invariant in the form of the second order tensor $\omega_{i j}$.

Next, we will assume that to the damage tensor $\omega_{i j}$, as an internal parameter of state, there corresponds its associated tensor $R_{i j}$ - a flow of thermodynamic stresses in damaged medium. For materials with an initial anisotropy, the equivalent stress $\sigma_{V}$ will be defined in terms of the simultaneous invariants of the stress tensor $\sigma_{i j}$ with the constitutive tensors $a_{i j}$ and $b_{i j k l}$ :

$$
\sigma_{V}=\sigma_{1}+\sigma_{2}, \quad \sigma_{1}=a_{i j} \sigma_{i j}, \quad \sigma_{2}=\sqrt{b_{i j k l} \sigma_{i j} \sigma_{k l}}
$$

Accordingly, we define the equivalent stress $\sigma_{* V}$ in terms of the simultaneous invariants of the stress tensor $\sigma_{i j}$ with the constitutive tensors $g_{i j}$ and $d_{i j k l}$ :

$$
\sigma_{*_{V}}=\sigma_{*_{1}}+\sigma_{*_{2}}, \quad \sigma_{*_{1}}=g_{i j} \sigma_{i j}, \quad \sigma_{*_{2}}=\sqrt{d_{i j k l} \sigma_{i j} \sigma_{k l}}
$$

Let us represent the dissipation potentials for creep and damage strain rates within the adopted assumptions

as

$$
\dot{D}=\dot{D}\left(\sigma_{V} ; \eta, T\right)=\sigma_{i j} \dot{c}_{i j}, \quad \dot{\Omega}=\dot{\Omega}\left(\sigma_{* V} ; \eta, T\right)=\sigma_{i j} \dot{\omega}_{i j}
$$

Then, by using the gradient law, the kinetic equations for creep and damage strain rates will be of the form:

$$
\dot{c}_{i j}=\dot{\lambda} \frac{\partial \dot{D}}{\partial \sigma_{i j}}, \quad \dot{\omega}_{i j}=\dot{\lambda}_{*} \frac{\partial \dot{\Omega}}{\partial R_{i j}},
$$

where $\dot{\lambda}$ and $\dot{\lambda}_{*}$ are the scalar factors that will be then determined from equalities (3) after specifying the type of each potential.

It is noteworthy that according to the basic thermodynamic inequality, we have:

$$
\dot{D}+\dot{\Omega} \geq 0, \quad \dot{D}\left(\sigma_{V} ; \eta, T\right) \geq 0, \quad \dot{\Omega}=\dot{\Omega}\left(\sigma_{*} ; \eta, T\right) \geq 0
$$

Let us establish a fracture criterion in the invariant scalar form. For this purpose, we consider the part of dissipation due to damage and its limiting value at the time instant after the hidden damage is completed: 


$$
\Omega(t)=\int_{0}^{t} \dot{\Omega} d t, \quad \Omega *=\int_{0}^{t_{*}} \dot{\Omega} d t
$$

Assume that the limiting value of the dissipation due to the creep damage of the material is a defined characteristic of the material. In this case, taking the quantity $\eta(t)=\Omega(t) / \Omega *$ as a scalar measure of energy for the fracture criterion, it can be written in the form of $\eta\left(t_{*}\right)=1$, where $t_{*}$ is the value of time at the instant of fracture.

Based on the thermodynamic assumptions for materials with an initial anisotropy in creep, we obtain the following constitutive equations:

$$
\dot{c}_{i j}=\frac{\dot{D}}{\sigma_{V}}\left(a_{i j}+\frac{b_{i j k l} \sigma_{k l}}{\sigma_{2}}\right), \quad \dot{\omega}_{i j}=\frac{\dot{\eta}}{R_{V}}\left(g_{i j} \frac{d_{i j k l} R_{k l}}{R_{2}}\right),
$$

where $c_{i j}$ and $\omega_{i j}$ are the symmetric tensors of irreversible creep and damage strains, $\dot{D}=\sigma_{i j} \dot{c}_{i j}$ is the power of dissipation due to creep, and $\dot{\eta}=R_{i j} \dot{\omega}_{i j}$ is the power of dissipation due to material damage referred to the limit value of dissipation $\Omega_{*}$ at the instant of fracture (the measure of damage, $0 \leq \eta \leq 1$ ).

Let us use the strain equivalence principle according to which, in order to establish the creep law in a damaged material, it is necessary to substitute the stress tensor in constitutive equations (7) for the creep strains in undamaged materials by the damage-associated stress tensor. The influence of the damage tensor on the effective stresses is taken into account using the following equalities: $R_{i j}=\sigma_{i j} /(1-\eta)$, with $\dot{\eta}=R_{i j} \dot{\omega}_{i j}(0 \leq \eta \leq 1), \eta\left(t_{*}\right)=1$.

Under the assumptions made and retention of the requirement of the basic thermodynamic inequality $\dot{D}+\dot{\Omega} \geq 0$, the dissipative potentials (3) may be written in the following way:

$$
\dot{D}\left(\sigma_{V} ; \eta, T\right)=\dot{D}\left(R_{V}\right), \quad \dot{\Omega}\left(\sigma_{* V} ; \eta, T\right)=\dot{\Omega}\left(R_{* V}\right),
$$

where $R_{V}=\sigma_{V} /(1-\eta)$ and $R_{*_{V}}=\sigma_{*_{V}} /(1-\eta)$ are the effective stress invariants.

Within the adopted assumptions, the principal directions of the material anisotropy are considered to be constant up to fracture, and the dissipation potential surfaces in the stress space extend in time in proportion to one parameter associated with the adopted measure of damage, $0 \leq \eta \leq 1$.

Consider constitutive equations (7) for transversely isotropic materials in creep. The dissipation potentials (8) will be specified in the form of power functions of stress invariants:

$$
\dot{D}=R_{V}^{N}, \quad \dot{\eta}=R_{* V}^{k} /(1-\eta)^{S},
$$

where $N, k$, and $S$ are the constants.

Equations (7) are considered as constitutive equations for materials with the initial anisotropy and asymmetry of creep and damage properties, in particular, in tension and compression. In the absence of the effects of the asymmetry of properties, we have $a_{i j}=g_{i j}=0$. In this case, for transversely isotropic materials, the constitutive equations of creep in the vector-matrix form taking into account the damage is written in the following way:

$$
\begin{array}{cc}
\underline{\dot{c}}=b_{1111}^{(N+1) / 2} \frac{\bar{\sigma}_{2}^{N-1}}{(1-\eta)^{N}}[B] \underline{\sigma}, \quad \underline{\dot{\omega}}=d_{1111}^{k / 2} \frac{\sigma_{* 2}^{k-2}}{(1-\eta)^{k+S-1}}[D] \underline{\sigma}, \\
\dot{\eta}=d_{1111}^{k / 2} \frac{\sigma_{* 2}^{k}}{(1-\eta)^{k+S}}, \quad \eta(0)=0, \quad \eta\left(t_{*}\right)=1,
\end{array}
$$


where $\underline{\dot{c}}=\left(\dot{c}_{11}, \dot{c}_{22}, 2 \dot{c}_{12}\right)^{T}, \underline{\sigma}=\left(\sigma_{11}, \sigma_{22}, \sigma_{12}\right)^{T}$, and $\underline{\dot{\omega}}=\left(\dot{\omega}_{11}, \dot{\omega}_{22}, 2 \dot{\omega}_{12}\right)^{T}$ are the creep strain rate, stress and rate vectors of the damage parameter, respectively, $\bar{\sigma}_{2}^{2}=\underline{\sigma}^{T}[B] \underline{\sigma}$ and $\sigma_{* 2}^{2}=\underline{\sigma}^{T}[D] \underline{\sigma}$ are the stress invariants,

$$
\begin{aligned}
& {[B]=\left|\begin{array}{ccc}
1 & \beta_{12} & 0 \\
\beta_{21} & \beta_{22} & 0 \\
0 & 0 & 4 \beta
\end{array}\right|, \quad \beta_{12}=-\frac{1}{2} b_{1111}, \quad \beta_{22}=\frac{b_{2222}}{b_{1111}}, \quad 4 \beta=\frac{b_{1212}}{b_{1111}},} \\
& {[D]=\left|\begin{array}{ccc}
1 & \delta_{12} & 0 \\
\delta_{21} & \delta_{22} & 0 \\
0 & 0 & 4 \delta
\end{array}\right|, \quad \delta_{12}=-\frac{1}{2} d_{1111}, \quad \delta_{22}=\frac{d_{2222}}{d_{1111}}, \quad 4 \delta=\frac{d_{1212}}{d_{111}} .}
\end{aligned}
$$

Next, we obtain the equations describing the influence of the cyclic loading on creep and damage for dynamic creep [13]. Assume that the stress tensor consists of the static component $\sigma_{0}$ and the rapidly varying component $\sigma_{1}: \sigma=\sigma_{0}+\sigma_{1}, \sigma_{1}=\sigma_{a} \sin (2 \pi f t)$, where $f$ is the frequency of forced vibrations, $f=1 / T, \sigma_{a}$ is the stress amplitude value. Using the previously described method [11] applied to the equations with a scalar damage parameter, we utilize the multiscale method and asymptotic small-parameter expansion of the primary unknowns $\mu=T / t$.

Applying this approach to Eqs. (10) and (11), we represent the processes of increase in creep strains and damage accumulation as the asymptotic expansions on two time scales (slow and fast $\xi=\tau / T$, where $\tau=t / \mu$ ):

$$
c \cong c^{0}(t)+\mu c^{1}(\xi), \quad \eta \cong \eta^{0}(t)+\mu \eta^{1}(\xi), \quad \omega \cong \omega^{0}(t)+\mu \omega^{1}(\xi),
$$

where $c^{0}(t), \eta^{0}(t), \omega^{0}(t), c^{1}(\xi), \eta^{1}(\xi)$, and $\omega^{1}(\xi)$ are the functions corresponding to the basic process of creep with damage in slow (0) and fast (1) time scales.

Taking into account the dependence of the creep strain, damage parameter and measure of damage on the slow time only [15], we obtain, after averaging

$$
\begin{cases}\left\langle c^{0}(\xi)\right\rangle=\int_{0}^{1} c^{0}(t) d \xi \cong c^{0}(t), & \left\langle c^{1}(\xi)\right\rangle=\int_{0}^{1} c^{1}(\xi) d \xi \cong 0, \\ \left\langle\eta^{0}(\xi)\right\rangle=\int_{0}^{1} \eta^{0}(t) d \xi \cong \eta^{0}(t), & \left\langle\eta^{1}(\xi)\right\rangle=\int_{0}^{1} \eta^{1}(\xi) d \xi \cong 0, \\ \left\langle\omega^{0}(\xi)\right\rangle=\int_{0}^{1} \omega^{0}(t) d \xi \cong \omega^{0}(t), \quad\left\langle\omega^{1}(\xi)\right\rangle=\int_{0}^{1} \omega^{1}(\xi) d \xi \cong 0 .\end{cases}
$$

Thus, using the asymptotic expansion method with subsequent averaging on the period of cyclic loading, in the case of a complex stress state, we derive the constitutive equations describing the dynamic creep with consideration of the damage in alloys with orthotropic properties:

$$
\begin{gathered}
\dot{\underline{c}}=b_{1111}^{(N+1) / 2} K\left(A_{n}\right) \frac{\bar{\sigma}_{2}^{N-1}}{(1-\eta)^{N}}[B] \underline{\sigma}, \quad \underline{\omega}=d_{1111}^{k / 2} H_{1}\left(A_{k}\right) \frac{\sigma_{* 2}^{k-2}}{(1-\eta)^{k+S-1}}[D] \underline{\sigma}, \\
\dot{\eta}=d_{1111}^{k / 2} H_{2}\left(A_{k}\right) \frac{\sigma_{* 2}^{k}}{(1-\eta)^{k+S}}, \quad \eta(0)=0, \quad \eta\left(t_{*}\right)=1,
\end{gathered}
$$


where

$$
\begin{gathered}
K\left(A_{n}\right)=\int_{0}^{1}\left(1+A_{n} \sin (2 \pi \xi)\right)^{N} d \xi, \quad H_{1}\left(A_{k}\right)=\int_{0}^{1}\left(1+A_{k} \sin (2 \pi \xi)\right)^{k-1} d \xi, \\
H_{2}\left(A_{k}\right)=\int_{0}^{1}\left(1+A_{k} \sin (2 \pi \xi)\right)^{k} d \xi, \quad A_{n}=\frac{\bar{\sigma}_{2}^{a}}{\bar{\sigma}_{2}}, \quad A_{r}=\frac{\sigma_{*_{2}}^{a}}{\sigma_{*_{2}}},
\end{gathered}
$$

$\left(\bar{\sigma}_{2}^{a}\right)^{2}=\left(\underline{\sigma}^{a}\right)^{T}[B] \underline{\sigma}^{a}$ and $\left(\sigma *_{2}^{a}\right)^{2}=\left(\underline{\sigma}^{a}\right)^{T}[D] \underline{\sigma}^{a}$ are the invariants of cyclic stresses.

Finite-Element Formulation of the Problem. Consider the statement of the two-dimensional problem in the theory of creep for a plane stress state. Let a body of volume $V$ made of a material with anisotropic creep and damage properties be fastened on the part of the surface $S_{1}$ and loaded by the surface forces $p\left\{p_{1}, p_{2}\right\}$ on the rest of the surface $S_{2}$. We will describe the planar motion of the continuum of material points in creep in the coordinate system $O X_{1} X_{2}$ within the Lagrangian approach. Let us introduce to the discussion the displacement vector of the points $u=\left\{u_{1}, u_{2}\right\}^{T}$ and their velocity $v=\left\{v_{1}, v_{2}\right\}^{T}$. According to these assumptions, the problem of creep for the case of small displacements and strains is described by the following initial boundary-value problem:

$$
\begin{gathered}
\sigma_{i j, j}+f_{i}=\rho \dot{v}_{j} \quad(i, j=1,2), \quad x_{1}, x_{2} \in V, \\
\sigma_{i j} n_{j}=p_{i}+p_{i}^{a} \sin 2 \pi f t, \quad x_{1}, x_{2} \in S_{2}, \\
v_{1}=d u_{1} / d t=\dot{u}_{1}, \quad v_{2}=d u_{2} / d t=\dot{u}_{2}, \\
\varepsilon_{11}=\frac{\partial u_{1}}{\partial x_{1}}, \quad \varepsilon_{22}=\frac{\partial u_{2}}{\partial x_{2}}, \quad \varepsilon_{12}=\frac{1}{2}\left(\frac{\partial u_{1}}{\partial x_{2}}+\frac{\partial u_{2}}{\partial x_{1}}\right) .
\end{gathered}
$$

Consider the case when stresses do not exceed the yield stress of the material. Assume that the components of the elastic strains $e_{i j}$ and creep strains $c_{i j}$ and also their rates are additive:

$$
\dot{\varepsilon}_{i j}=\dot{e}_{i j}+\dot{c}_{i j}
$$

The obtained system of equations (18)-(22) must be solved simultaneously with constitutive equations (16) and (17); in so doing, it is necessary to add the boundary conditions on the parts of the surface $S_{1}: \dot{u}_{i}=\dot{u}_{i}^{*}$ and $S_{2}$ : $\dot{\sigma}_{i j} n_{j}=\dot{p}_{i}$ and the initial conditions assuming that at the time instant $t=0$, the stress-strain state is defined by the solution of the elasticity theory problem.

System (16)-(22) is solved by using the previously described $[11,14]$ method of two time scales together with the averaging over the forced vibration period $1 / f$. Thus, the problem to be solved is formulated as a problem of static loading but with constitutive equations describing the dynamic creep.

The calculations employ the finite element method (FEM) with linear triangle elements. The resolving equation of the FEM formulated in terms of the rates is of the form

$$
[K] \underline{\dot{\delta}}=\underline{\dot{F}}+\underline{\dot{F}}_{c},
$$

where $[K]=\sum_{e} \int_{V^{e}}[B]^{T}[D][B] d V$ is the global matrix of the system, $\underline{\dot{F}}=\sum_{e} \int_{\Sigma_{2}^{e}}[N]^{T} \underline{\dot{p}} d \Sigma$ and $\underline{\dot{F}}_{c}=\sum_{e} \int_{V^{e}}[B]^{T} \underline{\sigma}^{*} d V$ are the vectors composing the global body force and fictitious load vector determined by creep strain. 


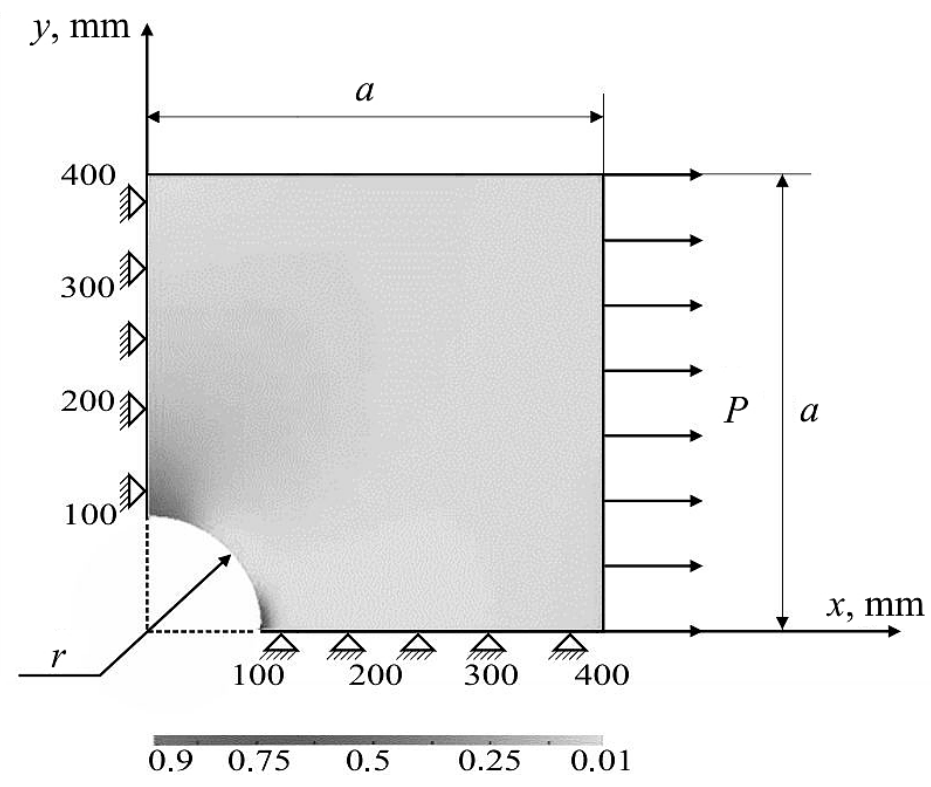

Fig. 1. Damage measure distribution $\eta=\eta(x, y)$ at $t=220 \mathrm{~h}$.

The software for computations was developed at the National Technical University "KhPI."

Anisotropic Creep and Damage in Plates. The developed method and software were used for simulation of creep and damage in titanium plates of alloy VT1-0 (alloys IMI125 and T40 are the analogs). The creep and creep rupture strength properties of the specimens cut out of a flat sheet in three directions, were experimentally obtained at the temperature $T=773 \mathrm{~K}$ in [14]. The values of the constants in Eqs. (16) and (17) obtained after experimental data processing are as follows: $k=N=5, s=1,\left(b_{1111}=2.303, b_{1122}=-1.151, b_{2222}=1.924, b_{1212}=2.058\right) \cdot 10^{-4}$ $(\mathrm{MPa})^{-2 N / N+1} /(\mathrm{h})^{2 / N+1},\left(d_{1111}=3.542, d_{1122}=-1.771, d_{2222}=3.324, d_{1212}=3.127\right) \cdot 10^{-5}(\mathrm{MPa})^{-2} /(\mathrm{h})^{2 / k}$.

To assess the validity of the results obtained, the comparison between the numerical computations and experimental data was performed under static creep up to fracture of the specimens cut out in various directions. The specimens were considered as long rectangular plates in tension. The discrepancy between numerical and experimental data does not exceed $25-32 \%$.

Next, the problems of static and dynamic creep of the titanium plate $(0.8 \times 0.8 \mathrm{~m})$ with a central hole of $0.1 \mathrm{~m}$ radius were solved. A quarter of the plate was considered, the stress strain state and damage variation with time were analyzed. Figure 1 illustrates the damage measure distribution for the case of static loading with a load of $10 \mathrm{MPa}$ at the time $t=220 \mathrm{~h}$ close to that to fracture $t_{*}=221 \mathrm{~h}$.

The calculations have resulted in the determination of the nonlinear dependence of creep and damage behavior on the load: thus, with increasing load from 10 to $13 \mathrm{MPa}$, the time to fracture decreases from 221 to $68.5 \mathrm{~h}$. Now, we present the dynamic creep simulation results for the same plate. In this case, the axial load consists of the static component $p_{0}=16.9 \mathrm{MPa}$ and the cyclic one, which varies according to the sinusoidal law at a frequency of $10 \mathrm{~Hz}$. We now introduce the loading parameter $L=p_{0} / p_{a}$, where $p_{a}$ is the amplitude value of the load. The variation of $L$ within the range of $0-0.3$ was considered.

The time to fracture for this scheme of loading decreases with increasing $L$ from $0.166 \mathrm{~h}$ for $L=0$ to $0.122 \mathrm{~h}$ for $L=0.3$. The character of the stress redistribution and the measure of damage is found to coincide qualitatively with those under static loading; however, a significant acceleration of the creep and after-effect processes due to dynamic creep takes place.

As a result of the calculations, it has been found that for low load values and, accordingly, for low values of stresses in the plate, the character of the creep process differs from that analyzed above. The dynamic load accelerates the stress relaxation in the region of the hole, as in the case of the dynamic creep [12], at which the stress level is so low that the damage accumulation rate decreases considerably. For example, for $p_{0}=13 \mathrm{MPa}$, the time to 
fracture $t_{*}$ for $L=0$ is $68.5 \mathrm{~h}$, but the addition of a small cyclic component $(L=0.05)$ results in the increase of the time to fracture $t_{*}=72.6 \mathrm{~h}$. The subsequent increase of $L$ leads to a similar increase of the time to fracture due to a fast dynamic relaxation at the beginning of the process: for $L=0.1$ we have $t_{*}=120.1 \mathrm{~h}$.

Conclusions. The method has been presented for solving dynamic creep and damage problems for structural elements made of materials with anisotropic properties. The use of the two time-scale method that is compatible with the asymptotic small parameter expansion and a subsequent averaging on a period, allows one to avoid the direct integration over the cycle and solve the problem only under the action of a static load, but with the derived constitutive equations of a special form. The effects of a significant acceleration of an increase in the strain and damage accumulation, as well as those of stress relaxation under dynamic creep, have been found for a plate with a hole, made of a titanium alloy with anisotropic creep and damage properties.

\section{REFERENCES}

1. N. R. Muzyka, "Influence of the anisotropy of sheet materials on the accuracy of measuring of the Vickers hardness," Strength Mater., 39, No. 2, 211-218 (2007).

2. M. Kuna, Finite Elements in Fracture Mechanics. Theory-Numerics-Applications, Springer, DordrechtNew York (2013).

3. S. Murakami, Continuum Damage Mechanics. A Continuum Mechanics Approach to the Analysis of Damage and Fracture, Springer, Dordrecht-London (2013).

4. G. Z. Voyiadis and P. I. Kattan, Damage Mechanics with Finite Elements: Practical Applications with Computer Tools, Springer (2012).

5. W. Zhang and Y. Cai, Continuum Damage Mechanics and Numerical Applications, Springer, Berlin (2010).

6. J.-F. Wen, S.-T. Tu, X.-L. Gao, and J. N. Reddy, "Simulations of creep crack growth in 316 stainless steel using a new creep-damage model," Eng. Fract. Mech., 98, 169-184 (2013).

7. E.-H. Kim, M.-S. Rim, I. Lee, and T.-K. Hwang, "Composite damage model based on continuum damage mechanics and low velocity impact analysis of composite plates," Comp. Struct., 95, 123-134 (2013).

8. L. Zhao, H. Jing, L. Xu, et al., "Numerical investigation of factors affecting creep damage accumulation in ASME P92 steel welded joint," Mater. Design, 34, 566-575 (2012).

9. L. V. Stepanova, "Refined calculation of the stress-strain state at the crack tip under cyclic loading in the medium with damage," Vestn. SamGU, Issue 2 (83), 105-115 (2011).

10. E. M. Adylina, S. A. Igonin, and L. V. Stepanova, "On a nonlinear eigenvalue problem resulting from the analysis of stresses at the fatigue crack tip," Vestn. SamGU, Issue 3/1 (94), 83-102 (2012).

11. V. I. Astaf'ev, Yu. N. Radaev, and L. V. Stepanova, Nonlinear Fracture Mechanics, "Samara University" Publishing House, Samara (2001).

12. D. Breslavsky and O. Morachkovsky, "Dynamic creep continuum damage mechanics: FEM-based design analysis," in: Computational Plasticity: Fundamentals and Applications (Proc. of the Fifth Int. Conf. on Computational Plasticity, March, 17-20, 1997, Barcelona), Pt. 1, IMNE, Barcelona (1997), pp. 1071-1076.

13. S. Taira and R. Otani, Theory of High-Temperature Strength of Materials [Russian translation from Japanese], Metallurgiya, Moscow (1986).

14. V. N. Konkin and O. K. Morachkovskii, "Creep and long-term strength of light alloys with anisotropic properties," Strength Mater., 19, No. 5, 626-631 (1987).

15. D. Breslavsky and O. Morachkovsky, "A new model of nonlinear dynamic creep," in: IUTAM Symp. on Anisotropy, Inhomogeneity and Nonlinearity in Solid Mechanics, Kluwer Academic Publishers, Dordrecht (1995), pp. 161-166. 